\title{
Capital social en empresas familiares
}

\author{
Jiménez, Magda \\ Piña Zambrano, Henri
}

\section{Resumen}

Este artículo está dirigido a caracterizar la dinámica de la gestión del capital social en empresas familiares ubicadas en Santa Ana de Coro, municipio Miranda del estado Falcón. Para ello se llevó a cabo un estudio de campo de tipo descriptivo, mediante el uso de la técnica de la encuesta, siendo la modalidad utilizada el cuestionario, con el interés de obtener datos e información directamente de las 08 empresas familiares que funcionan en este municipio. De esta manera se conoció que en las empresas familiares del municipio Miranda, el capital social representa significativamente una de las características conducentes a la sociabilidad y confianza entre los miembros de las familias conductoras de las organizaciones y los empleados no miembros de familia; y es precisamente esta dinámica la razón por la cual el capital social se convierte en un trascendental recurso para las empresas, permitiendo el acceso a otros recursos e impulsando la participación de todos los que hacen vida en la organización, y por ende el crecimiento y desarrollo económico se hace evidente, estimulando en los empleados el sentido de pertenencia, confianza, compromiso y reciprocidad, generando beneficios para la red de demandantes y consumidores.

Palabras clave: Capital social, empresa familiar, Falcón, emprendimiento.

\section{Social Capital in Family Enterprises}

\begin{abstract}
This article is directed toward characterizing the social capital management dynamic in family enterprises located in Santa Ana de Coro, Miranda Municipality, State of Falcon. To accomplish this, a field study of the descriptive type was carried out using the survey technique with a questionnaire to obtain data and information directly from the 8 family businesses functioning in this municipality. It became known that in family businesses of the Miranda Municipality, social capital represents signifi-

\section{Recibido: 17-11-10. Aceptado: 06-05-11}

* Licenciada en educación y en desarrollo empresarial, egresada del programa de desarrollo empresarial de la Universidad Francisco de Miranda. E-mail: helysquintero@hotmail.com

** Ingeniero Agrónomo. MSc. en gerencia agrícola. Doctorando en desarrollo rural (Universidad de Córdoba, España). Profesor del departamento de desarrollo y producción agrícola. Universidad Francisco de Miranda, Falcón, Venezuela. E-mail: henripina@gmail.com; henripina@ unefm.edu.ve
\end{abstract}


Capital social en empresas familiares

Jiménez, Magda y Piña Zambrano, Henri

cantly one of the characteristics leading to sociability and confidence among members of the families that lead the organizations and non-family member employees. This dynamic is precisely the reason that social capital becomes a transcendental business resource, permitting access to other resources, impelling participation of all those who earn a livelihood in the organization; therefore, growth and economic development become evident, stimulating the employees' sense of belonging, confidence, commitment and reciprocity, and generating benefits for the network of applicants and consumers.

Key words: Social capital, family business, Falcon, entrepreneurship.

\section{Introducción}

Las organizaciones empresariales han representado para el desarrollo económico, un alto potencial e impulso laboral, así como un gran estímulo para la creación y crecimiento de las mismas desde un punto de vista social y familiar, aplicado este último a la sucesión de bienes, dedicación y compromiso asumidos por miembros de una familia donde el sentido de pertenencia juega un rol importante para éstos, ya que al unir esfuerzos, la empresa se hace más competitiva.

En este sentido, en las empresas familiares lo más común es que el capital sea de un cónyuge y con el tiempo, también los hijos entren a formar parte de la compañía, no obstante puede haber una venta a terceros. Sin embargo, en la mayoría de las empresas familiares, su creación persigue la permanencia en el tiempo como una organización que aplica valores y fortalece su constitución en el ámbito familiar, permitiéndole alcanzar el éxito desde esta formación (Durston, 2002).

En este tipo de empresas, el capital social representa un aspecto gerencial fundamental, visto éste como un activo intangible, aplicable para determinar desde la correcta ocupación de cargos de dirección y gerencia, hasta cómo se conducen las relaciones interpersonales hacia el éxito y a una asociatividad cónsona con sus fines y metas, en la búsqueda de competitividad y consolidación en el mercado.

De manera general, las empresas exitosas disponen de un marco filosófico estratégico, una clara dirección gerencial y sistemas de información modernos a través del cual pueden medir su efectividad y competitividad, atendiendo a parámetros e indicadores, facilitando su control y funcionamiento mediante las actividades de gestión financiera, operativa y administrativa propias de la empresa.

De acuerdo con este planteamiento, la gestión empresarial, basada en indicadores, induce la eficiencia, eficacia y productividad de cada departamento, permitiendo priorizar actividades y procesos para dar cumplimiento a objetivos de corto, mediano y largo plazo. Asimismo conducen a identificar oportunidades de mejoramiento y optimización en aquellas funciones o procesos que por su proceder y tendencia, requieren una reorientación o refuerzo para mejorar cualquier situación de la organización, sin importar su tamaño o figura jurídica (Vainrub, 2006).

El señalamiento anterior también es aplicado a las empresas familiares, las cuales en los últimos años, han adquirido un importante nivel de complejidad. Consecuentemente, se observa la necesidad por parte de éstas de trasmitir una ima- 
gen de marca definida para comunicar y difundir mensajes específicos sobre aquellos atributos que le distinguen y posicionan en el mercado. En términos concretos, la empresa familiar corresponde a un tipo de iniciativa económica cuya propiedad está en manos, total o mayoritariamente, de uno o varios miembros de una misma familia (Vainrub, 2006).

No obstante, ellas llevan implícitas profundos valores y anhelos, junto a un alto grado de lealtad y apoyo a las políticas adelantadas por la empresa, por lo tanto, enfrentan los conflictos internos y externos, toleran crisis, porque sienten que abandonar el negocio, es abandonar la familia y por estas causas permanecen en el tiempo, a diferencia de otro tipo de empresas.

Es así como las empresas familiares están signadas por rasgos culturales distintivos, siendo necesario su estudio en atención a sus interconexiones económicas integradas en relaciones, redes y estructuras sociales. Es necesario incluir más dimensiones socioculturales en los llamados estudios económicos administrativos de las empresas familiares y dejar de lado el manejo de lenguaje abstraído, descontextualizado y funcionalista, el cual se convierte en un obstáculo que no permite ver la complejidad social existente en torno de este tipo de empresas (Contreras y Regalado, 2009).

Es allí donde resalta el capital social como un activo intangible, manifestado en la capacidad de confianza (entendida, como el clima prevaleciente para realizar negocios), valores cívicos (representativos de ciertas actitudes, como la educación en el seno de las familias y la ética hacia el trabajo) y aso- ciatividad que pueda lograr la sociedad (Kliksberg, 2001).

El capital social surge entonces, dada la asociación familiar para emprender una iniciativa de negocio y hacerlo productivo, de la tradición en la cooperación, la solidaridad, la confianza, junto al establecimiento de acuerdos entre los socios (López y Contreras, 2009).

En términos concretos, el capital social es un concepto que ha resurgido en respuesta a los fracasos de las ideas economicistas del desarrollo, resaltando la necesidad de incluir y rejerarquizar en este proceso, valores como la confianza interpersonal, la asociatividad, la conciencia cívica, la ética y los valores predominantes en la cultura de una sociedad (Moreno, 2005).

Las empresas familiares, por lo general adoptan los mismos indicadores de gestión que otras y no prestan la atención necesaria a los de gestión del capital social, los cuales son fundamentales para mantener un clima de armonía dentro de la organización, atenuando los distintos conflictos que generalmente se presentan entre los familiares, vinculados fundamentalmente a la toma de decisiones y la cadena de mando dentro de la organización.

En este sentido, cuando familiares cercanos trabajan juntos, a menudo las emociones interfieren con las decisiones de negocios. En ciertas empresas familiares, el control de las operaciones diarias es un problema. En otras sufren porque algunos de los familiares no desean reinvertir las ganancias en la empresa, lo cual impide el crecimiento del negocio (Vainrub, 2006). 
Capital social en empresas familiares

Jiménez, Magda y Piña Zambrano, Henri

Así, son numerosos los problemas presentados en una empresa familiar, así como también numerosas las estrategias gerenciales para solventarlas; sin embargo, este estudio está referido a un tema poco abordado por investigadores y estudiosos de la materia, tal como es la gestión del capital social en empresas familiares, abordando específicamente aquellas ubicadas en la categoría de supermercados localizados en el municipio Miranda, Falcón.

En este contexto, se conciben a las empresas familiares como entidades dinámicas, un sistema de tres círculos (empresa, familia y gerencia) involucrados entre sí, las cuales a medida que maduran y crecen, el número de personas involucradas en cada uno de ellos, aumenta y, con ello, se potencia el grado de complejidad en cuanto a sus relaciones. Es ésta, la razón por la cual este tipo de organización se están consolidando y evolucionando dentro del mercado comercial.

La vigencia del componente familiar en muchas empresas, se debe a que su objetivo principal es dar trabajo a los miembros de la familia, antes o después de ser accionistas. En este contexto, la estructura empresarial debe contener, en términos laborales, las necesidades de accionistas y familiares, en especial en momentos de crisis. Esta línea también tiene el objeto de mantener unida la familia, edificando tradición, memoria e historia común.

Otro aspecto está referido al aporte social, al considerar el capital social en la gestión empresarial como un indicador de competitividad orientado a subsanar la relación entre el sistema familia-empresa, con la finalidad de poder identificar los principales problemas y conflictos inherentes a la organización, desarrollar modelos donde se distingan los factores y relaciones que marcan los efectos tanto positivos como negativos, correlacionándolos con indicadores de éxito y fracaso del desarrollo empresarial.

En este sentido, los aportes a las empresas familiares se distinguen en el hecho de que al concebir los elementos del capital social como un indicador para subsanar situaciones particulares familia-empresa, permitirá de acuerdo con el compromiso asumido, una visión amplia de las estrategias a largo plazo para el desarrollo competitivo de la empresa y de esta manera, mantener el legado en el tiempo. Además, el compromiso es extendido a empleados no miembros de la familia, cuando son asumidos con responsabilidad por familiares y conllevan a la lealtad de generación en generación (Vainrub, 2006).

Por otro lado, la confianza y la capacidad para resguardar el conocimiento por miembros de la familia, permite a los nuevos miembros, adquirir las experiencias gerenciales y estratégicas bajo las cuales se ha formado y crecido la empresa. Esto en cierta forma permite que los sucesores conozcan a profundidad la misma, generando confianza entre los empleados, proveedores y miembros de la familia; y por ende un amplio sentido de pertenencia por la organización (Vainrub, 2006).

De manera específica, en el estado Falcón existen muchas familias que han establecido empresas familiares, generando una serie de relaciones, redes y grupos sociales, mecanismos de comunicación, participación política e inclusión social, todo ello basado en los 
elementos de confianza, reciprocidad y cooperación.

En este contexto se desarrolló una investigación dirigida a caracterizar la dinámica de la gestión del capital social en las empresas familiares del sector supermercados localizados en municipio Miranda, estado Falcón, enfatizando por un lado la dinámica de la gestión del capital social y por otro, los criterios de gestión empresarial aplicados por dichas empresas.

Este tema no ha sido estudiado ampliamente en el país y aún menos en el estado, por lo tanto constituye por un lado, un aporte científico dado su carácter pionero, pues existen muchos antecedentes de investigaciones relacionadas con indicadores de gestión empresarial, pero ninguno referido a la gestión del capital social en empresas familiares. Así mismo, el estudio suministra nuevos aportes al conocimiento de las ciencias sociales y se convierte por tanto, en un referencial de consulta para futuras investigaciones en éste tema o para estudios de aplicabilidad práctica en alguna empresa familiar.

En términos concretos, el estudio correspondió a una investigación de campo, de tipo descriptiva en la cual se entrevistaron 8 empresas familiares del sector supermercado, localizadas en municipio Miranda, estado Falcón (Cuadro 1).
El análisis de la data se soportó en estadísticas descriptivas a través de la elaboración de cuadros de frecuencias y porcentajes, así como en la elaboración de categorías de análisis, construidas a partir de la información suministrada por los sujetos de estudio.

Por otro lado, debe destacarse una limitante de suma importancia durante el desarrollo de la investigación, la cual impidió obtener una mayor cantidad de datos para profundizar en el análisis del estudio, tal como lo es el difícil acceso a la información. De las ocho (08) empresas objeto de estudio sólo se logró la entrevista y respuestas satisfactorias al cuestionario de cuatro (04), las otras empresas no suministraron información alegando ocupaciones laborales y poca disposición para atención investigador. Esta es una de los principales rasgos característicos de las empresas familiares, como lo es el celo a difundir información acerca de su desempeño y manejo gerencial.

\section{Capital social}

Es ya ampliamente aceptada la importancia de las relaciones y las actividades sociales en el desempeño económico, al servir de elemento promotor y facilitador para la generación e intercam-

\section{Cuadro1}

Ficha técnica de la investigación

\begin{tabular}{ll}
\hline Universo de estudio & 8 empresas familiares del sector supermercado \\
Sujeto de estudio & Director - gerente de cada supermercado \\
Procedimiento de investigación & Entrevista semiestructurada a partir de un cuestionario base \\
Ámbito geográfico & Municipio Miranda, estado Falcón \\
Período de análisis & Marzo - agosto de 2010 \\
\hline
\end{tabular}

Fuente: Elaboración propia. 
Capital social en empresas familiares

Jiménez, Magda y Piña Zambrano, Henri

bio de transacciones (sociales y económicas) entre individuos. Los académicos han llamado de manera genérica a este fenómeno, capital social.

Aunque la profundización en la discusión del concepto es reciente, hace aproximadamente 20 años, la literatura señala como primeras referencias al término, el arte asociativo de los norteamericanos (Tocqueville, 1854) o más recientemente, el papel de normas de reciprocidad y redes sociales en el compromiso comunitario (Hanifan, 1916). Sin embargo, el auge científico sobre el tema ha sido más significativo en las últimas décadas (Forsman, 2005).

Es precisamente lo novel del tema, una de las principales razones por las cuales se le atribuye su carácter ambiguo y ese efecto paraguas bajo el cual se colocan diversos temas para explicar diversos comportamientos: todo es causa y efecto. Así, un elemento con fuerte influencia en el debate y controversia en torno al capital social lo representa, precisamente el término capital, el cual enfocado desde diferentes disciplinas, es analizado en función de tales concepciones.

De esta manera, posiciones sociológicas o económicas, difieren significativamente en el uso y construcción de postulados y abordaje empírico de casos de estudio. Sin embargo, debe tenerse presente la ubicación del término capital como categoría económica, por tanto se asume una concepción de uso, reproducción y acumulación de recursos.

En este estudio, se considera al capital social como capital, en tanto proporciona beneficios a quienes lo poseen. Es un factor capaz de acumularse y reprodu- cirse. De esta manera, se ubica al concepto en el plano conductual de las relaciones y los sistemas sociales. Igualmente es recurso, y vía para acceder a los recursos, el cual en combinación con otros factores, gestiona beneficios para quienes lo poseen (Durston, 2002).

Este planteamiento hace referencia a la relación entre individuos, humana, basada en el principio de reciprocidad y sistema de intercambio, entre estos. El sistema de compensaciones no es obligatorio, no es inmediato, ni es equivalente. Son compensaciones humanas derivadas de relaciones sociales producto de interacciones pasadas con fuerte determinación histórica y con perspectivas de largo plazo, constituyéndose así en la base fundamental de las instituciones del capital social (Durston, 2002).

Bajo esta concepción, el capital social se transforma en un importante recurso, herramienta en la estrategia de vida de los individuos al permitirle el acceso a otros recursos, fomentar su participación en organizaciones y promover cursos de acción orientados a mejorar su nivel de vida e inclusión social (Arriagada, 2006). Partiendo de estos planteamientos, se identifican tres aportes fundamentales al estudio del capital social.

Un primer aporte concibe al capital social como aspectos de las organizaciones sociales (redes, normas y confianza), los cuales facilitan la acción y la cooperación para el beneficio mutuo de los individuos (Putnam, 1995). El capital social reside en las asociaciones creadas por el hombre, como mecanismos para regular el funcionamiento y desempeño de actividades individuales y (o) colectivas, deviene de redes o contactos de personas o 
entre grupos de personas quienes hacen funcionar la sociedad.

Este aporte caracteriza a su vez al capital social como un compromiso cívico, reciprocidad, confianza y asociatividad de donde surge el beneficio, para quienes forman la red o para quienes sin pertenecer a esta, disfrutan de tales beneficios. Esta concepción del capital social no enfatiza la existencia o no de lazos sociales, lo cual se da por presente, por el contrario, se focaliza en los vínculos horizontales y recíprocos, junto a relaciones verticales para acceder a diferentes tipos de recursos (Putnam, 2004).

De esta manera, una sociedad con significativa presencia de valores de capital social, se corresponde a un ambiente propicio para la confiabilidad de relaciones y se favorecen flujos de información, derivando así en el nacimiento de normas consensuadas y aceptadas por todos por igual (Putnam, 2003).

Otro abordaje teórico considera este término como la socioestructura sobre la cual se constituye un activo de capital para quien lo posee, facilitándole ciertas acciones al poseerlo. Por tanto, el capital social es un factor productivo en correspondencia con la capacidad intrínseca de la persona para trabajar (cooperar) en grupos con otros individuos dentro de un marco rector de normas y valores aceptados en conjunto (Coleman, 1990).

Este enfoque del capital social centra su atención, no en la naturaleza de las relaciones sociales, sino en la estructura funcional de la red conformada por los individuos, destacando los beneficios que estos pueden obtener al participar en dicha red, ubicando la esencia del capital social en un contexto individual: es la per- sona quien posee los contactos en una red, para alcanzar sus objetivos particulares bajo ciertos parámetros de conducta y comportamiento (Coleman, 1992).

Igualmente se caracteriza por el origen propio de la red (proviene de ella) y una expectativa de beneficio mutuo (individual o colectivo) basado en un sistema de reciprocidad y confianza, dando origen al marco regulador (en ocasiones, tácito) de sanciones y premios para los individuos de la red (Coleman, 1986).

Un tercer planteamiento considera al capital social como el agregado de recursos reales o potenciales ligados a la posesión de una red durable de relaciones cuasi institucionalizadas de reconocimiento mutuo. En este sentido, las relaciones sociales son en sí mismas el capital social del individuo, quien puede hacer uso para acceder a recursos, de aquellos otros individuos con quienes está en contacto a través de esas redes (Bourdieu, 1985).

Según este enfoque, el capital social consta de dos elementos básicos: la relación social propiamente dicha, manifestada en los encuentros de los individuos con actores sociales (reuniones, eventos o núcleo familiar) y el beneficio obtenidos por los individuos (recursos o accesos a recursos) derivados de esta relaciones sociales, tanto en calidad como cantidad de estos beneficios (Bourdieu, 1989). Estos son los tres principales aportes al estudio y análisis del capital social (Cuadro 2).

Existen otros estudios de significativa importancia, pero se consideran derivaciones de estas primeras concepciones. Así al explicar el funcionamiento del capital social, se hace mención a la naturaleza gregaria del ser humano: los individuos no son entes aislados, por el contrario, mantie- 
Capital social en empresas familiares

Jiménez, Magda y Piña Zambrano, Henri

\section{Cuadro 2}

Principales ideas y definiciones de capital social

\begin{tabular}{|c|c|c|c|}
\hline Autor & Definición de Capital Social & Variables Claves & Énfasis del Concepto \\
\hline PUTNAM & $\begin{array}{l}\text { Aspectos relacionados con } \\
\text { las organizaciones sociales } \\
\text { (redes), normas y confianza; } \\
\text { las cuales facilitan la coordi- } \\
\text { nación y cooperación para el } \\
\text { beneficio mutuo. }\end{array}$ & $\begin{array}{l}\text { - Intensidad de la aso- } \\
\text { ciación horizontal. } \\
\text { - Participación activa de } \\
\text { ciudadanos en actos } \\
\text { públicos. }\end{array}$ & $\begin{array}{l}\text { Dimensión política pre- } \\
\text { valece sobre la econó- } \\
\text { mica. Las condiciones } \\
\text { cívicas de una comuni- } \\
\text { dad determinan su de- } \\
\text { sempeño institucional. }\end{array}$ \\
\hline COLEMAN & $\begin{array}{l}\text { No es una entidad simple, } \\
\text { sino por el contrario es una } \\
\text { variedad de entidades con } \\
\text { dos características funda- } \\
\text { mentales: estas entidades } \\
\text { son una forma de estructura } \\
\text { social y facilitan las acciones } \\
\text { de aquellos individuos quie- } \\
\text { nes pertenecen a dichas enti- } \\
\text { dades. }\end{array}$ & $\begin{array}{l}\text { - Sistemas familiares y } \\
\text { educativos. } \\
\text { - Organizaciones verti- } \\
\text { cales y horizontales. }\end{array}$ & $\begin{array}{l}\text { Las acciones individua- } \\
\text { les determinan el inter- } \\
\text { cambio en la sociedad. }\end{array}$ \\
\hline BOURDIEU & $\begin{array}{l}\text { Conjunto de recursos (real o } \\
\text { potencial) derivado de perte- } \\
\text { necer institucionalmente por } \\
\text { un significativo período de } \\
\text { tiempo a redes de conocimien- } \\
\text { to o reconocimiento mutuo. }\end{array}$ & $\begin{array}{l}\text { - Duración y amplitud } \\
\text { de las relaciones. } \\
\text { - Conexiones efectivas } \\
\text { de la red con otras re- } \\
\text { des. }\end{array}$ & $\begin{array}{l}\text { La naturaleza histórica } \\
\text { de relaciones y su pro- } \\
\text { yección en la sociedad y } \\
\text { en los medios de produc- } \\
\text { ción. }\end{array}$ \\
\hline
\end{tabular}

Fuente: Milani, 2003; Puente \& Torrealba, 2003.

nen interconexiones económicas integradas en relaciones, redes y estructuras sociales (Granovetter, 1973).

Derivada de estas interconexiones, forma parte del capital social en la medida que la racionalidad de objetivos de los individuos, las relaciones sociales establecidas por éstos para alcanzarlos, constituyen sus activos económicos y de los grupos de los cuales forman parte (Granovetter, 1985). Igualmente se utiliza el capital social para explicar el desarrollo y crecimiento de un país. Se recurre a normas y valores compartidos por los individuos, en tanto, la sociedad, para promover la confianza y la cooperación.
En este sentido, también se considera que el capital social corresponde a una estrategia para valorar el comportamiento cultural dentro de un proceso de desarrollo, atendiendo a la diferencia entre culturas para promover el crecimiento y el desarrollo (Fukuyama, 1999).

Partiendo de estos planteamientos sobre capital social, se distinguen elementos comunes del término, los cuales en definitiva son los mecanismos y procesos básicos los cuales actúan y sustentan todo el andamiaje del capital social. Entre estos se pueden mencionar: confianza, reciprocidad y cooperación (Cuadro 3) (Durston, 2002). 
Cuadro 3

Elementos comunes y distintivos del capital social

\begin{tabular}{ll}
\hline Elemento & \multicolumn{1}{c}{ Descripción } \\
\hline Confianza & $\begin{array}{l}\text { Comportamiento esperado de otra persona (contraparte) con quien se ini- } \\
\text { cia o mantiene una relación soportada en un mecanismo de reciprocidad y } \\
\text { elementos afectivos, derivada de repetidas interacciones con dicha perso- } \\
\text { na. La confianza implica ceder parte del control de bienes propios a la otra } \\
\text { persona con quien se mantiene (o inicia) la diada. } \\
\text { La confianza deviene, tanto de normas generalizadas de moralidad, como } \\
\text { de fuentes personalizadas de integración social. Los individuos no nece- } \\
\text { sariamente buscan en todas las oportunidades maximizar los beneficios, } \\
\text { consideran en cambio, minimizar en cada transacción el riesgo y maximi- } \\
\text { zar el acceso a recursos a través del establecimiento de relaciones con } \\
\text { otros individuo (Lyon, 2000). } \\
\text { Principio fundamental rector de relaciones institucionalizadas, formales e } \\
\text { informales, entre individuos; sustentadas en la lógica de intercambio de re- } \\
\text { tribuciones. Una retribución implica la disposición a iniciar o mantener una } \\
\text { relación social y la obligación cultural de quien recibe, de retribuir en cierta } \\
\text { forma, la retribución recibida, a riesgo de la sanción moral (tácita o explíci- } \\
\text { ta) de la persona con quien mantiene la relación. } \\
\text { Acción complementaria orientada al logro de objetivos compartidos en un } \\
\text { emprendimiento común y fortalecida mediante la repetición de situaciones } \\
\text { de confianza. }\end{array}$ \\
\hline Cooperación & \\
\hline &
\end{tabular}

Fuente: Durston, 2002.

Estos tres elementos conforman el sustento del mecanismo del capital social, esquematizado como un flujo lineal de retroalimentación: partiendo de la confianza surgida entre dos individuos, alimentada constantemente por un mecanismo de reciprocidad, deriva un proceso de cooperación en torno a objetivos y metas comunes. Se institucionaliza así un comportamiento, primero individual y luego colectivo, a partir del cual surgen las normas y patrones de comportamiento rectores de los comportamientos de la sociedad en su conjunto, siendo el comportamiento de la sociedad el resultado de la sumatoria de los comportamientos individuales de sus ciudadanos.

\section{Empresas familiares}

Se considera una empresa familiar aquella unidad económica cuya propiedad está en manos de una o varias familias, cuyos miembros intervienen de forma decisiva en la administración y dirección del negocio (Vainrub, 2006; Ministerio de Economía, 2003). Existe, por tanto, una estrecha relación entre propiedad y gestión o, dicho de otro modo, entre la vida de la empresa y la vida de la familia.

En este sentido, se considera como valores presentes en la empresa familiar, los siguientes (Ministerio de Economía, 2003:19): 
Capital social en empresas familiares

Jiménez, Magda y Piña Zambrano, Henri

a) Mayor grado de compromiso y dedicación con el negocio. El empresario arriesga, de forma permanente, el patrimonio, el prestigio y el futuro de su familia. Necesita el éxito y extrema su empeño en lograrlo.

b) Mayor grado de autofinanciación y reinversión de beneficios para financiar el futuro crecimiento de la empresa. Ello conlleva, asimismo, un control más riguroso del gasto.

c) Mejor orientación al mercado. Las empresas familiares, generalmente, tienen sus formas propias de hacer las cosas, un know how comercial que no poseen sus competidores y que se transmite a las siguientes generaciones, perpetuándolo como estrategia de dirección.

d) Mayor vinculación con los clientes. El compromiso que caracteriza a las empresas familiares suele manifestarse bajo la forma de una atención más cordial y esmerada y una calidad de servicio más alta, dando origen a un mayor grado de confianza y reputación entre los clientes.

e) Comportamiento más entusiasta de los trabajadores. Generalmente el fundador tiene una mejor relación y siente una mayor responsabilidad hacia sus trabajadores, quienes le han ayudado al inicio de la empresa, traduciéndose en una buena valoración de éstos hacia él y en sentirse que forman parte de un equipo.

f) Flexibilidad competitiva y capacidad de ajuste en las fases depresivas del ciclo económico, con efectos notables sobre la estabilidad del empleo.

\section{Capital social y empresas familiares}

\subsection{Características generales de la gestión empresarial en empresas familiares}

En términos generales, los elementos del capital social (Durston, 2002) se enfocan en la confianza, reciprocidad y cooperación; ahora bien al profundizar en la especificidad de las empresas familiares y fundamentalmente en la gestión de las mismas, estos elementos toman un carácter gerencial (Beltrán, 2004), por lo cual se enfatizan los procesos desde el nivel estratégico hasta el nivel operativo, asociados a los objetivos planificados en la organización, concebidos sobre una empresa en funcionamiento y basados en información proactiva mediante la toma efectiva de decisiones. En este caso, el enfoque se centró en la organización administrativa de la empresa.

Sobre este particular se tiene que el $75 \%$ de las empresas están divididas por departamentos, considerado por los gerentes de las mismas, como una manera eficiente de organizarlas y mantener su control a través de la comunicación directa con los responsables de estas áreas. El restante $25 \%$ considera la centralización, es decir, manejar la organización desde la gerencia y organizar la misma de acuerdo con la dinámica y exigencias del mercado.

Por otro lado, la totalidad de la asignación de actividades y funciones de las empresas, se hace de acuerdo al cargo desempeñado por los empleados en 
cada uno de sus departamentos. Este esquema de organización le permite a la gerencia mantener un mayor control sobre las acciones a emprender. Se diseña una estructura adaptada a las necesidades y requerimientos exigidos, proyectada siempre al cumplimiento de su misión y visión.

En este sentido, el principio de departamentalización favorece agrupar a los empleados y las actividades en secciones, facilitando la expansión de la organización. Los argumentos usados frecuentemente para determinar la estructura departamental, se basan en la división efectiva del trabajo (Flores, 1999). En términos generales, la mayoría de las empresas familiares consideran este principio como forma de establecer el funcionamiento, control y dirección de su empresa.

Todas las empresas consultadas realizan sus planificaciones según las necesidades surgidas durante su desarrollo y crecimiento, aunque sólo $50 \%$ planifican en conjunto: gerencia y supervisores. El otro $50 \%$ considera que sólo la gerencia debe realizar las estrategias de planificación, ya que es directamente ésta instancia la responsable de conocer y manejar el funcionamiento de la organización para posteriormente bajar las directrices y decisiones a los subordinados.

Las principales áreas estratégicas abordadas, son: ventas, compras, promociones de proveedores, propaganda y publicidad. En cuanto al tiempo para planificar, $25 \%$ planifica trimestralmente, en reuniones formales con gerentes $y$ supervisores, donde se manifiestan las debilidades y se escuchan propuestas para mejorar la estrategia de ser necesario. Otro $25 \%$ realiza sus planificaciones bimensualmente con la intención de constantemente estar vigilantes ante el desarrollo de las ventas y crecimiento económico de la empresa, además de verificar el desempeño de los trabajadores y su compromiso con la organización. El restante $50 \%$, planifica de acuerdo a las necesidades diarias de la empresa.

La planificación estratégica, es uno de los aspectos de suma importancia para el funcionamiento de una organización, para ello se debe centrar la atención en la misma y realizarla en función de su misión, visión y objetivos. Sobre este particular, la literatura refiere como principales áreas a las cuales los directivos de empresas prestan mayor atención, las ventas, producción, servicio al cliente y la calidad (Pérez, 2004).

Además de las actividades de planeamiento estratégico, otras áreas como la investigación y el desarrollo, ocupan un lugar secundario en la atención de los altos directivos. En un lugar intermedio se encuentra el área de aprovisionamiento y logística. En este sentido las empresas deben perseguir una estrategia de especialización orientada a afianzar o expandir la presencia de sus productos en el mercado (Pérez, 2004). De esta manera, la conducción de la empresa familiar debe orientarse a desarrollar en su seno, un consenso claro y explícito sobre los objetivos a perseguir

Por otro lado, en los esquemas jerárquicos en las empresas familiares aparece como presidente o gerente general, habitualmente al fundador. Sólo se evidenciaron dos casos donde este cargo lo ostentaba un tío y un grupo de hermanos, respectivamente. En la segunda línea de mando, aparece la madre 
Capital social en empresas familiares

Jiménez, Magda y Piña Zambrano, Henri

/ esposa del padre o el hijo. En una tercera línea de mando están los hermanos, esposas o hijos.

Para las empresas familiares, mantener esta figura implica necesariamente que miembros de la familia formen parte de la dirección y control, así como también ser partícipes directos de las decisiones, planificación de estrategias, resolución de conflictos familiares y mantener en vigencia la misión que la familia se propuso una vez creada la empresa.

Sin embargo, conviene distinguir que en una empresa familiar dirigida por la primera generación es normal que tanto las responsabilidades de gobierno como los de alta dirección sean desempeñadas por una o pocas personas, en este caso, los fundadores. En este caso, el gobierno es entendido como el conjunto de mecanismos desarrollados para operar una empresa con el objeto de proteger los intereses, las acciones los derechos y obligaciones de quienes tienen interés legítimo en ella. Estos como únicos propietarios, mantienen el control de la mayoría de las decisiones (Vainrub, 2006).

Dentro de este marco debe considerarse la existencia de riesgos frente a la posibilidad de incompatibilidad de las responsabilidades de gobiernos y de dirección, puesto que puede reinar un clima emocional o excesiva preferencia personal por los fundadores en la dirección estratégica. Por tanto, cuando la empresa familiar llega a un número significativo de directivos, es necesario un mecanismo idóneo para dirigir la organización hacia metas orientadas a resultados y beneficios eficaces basados en una planificación desde el punto de vista económico y financiero a mediano y largo plazo.

\subsection{Elementos del capital social presentes en las empresas familiares}

Tal como se mencionó, a los fines de la presente investigación, se considera al capital social como un recurso intangible, que permite a personas y grupos obtener beneficios por medio de relaciones sociales dotadas de confianza, reciprocidad y cooperación. Dentro de éste contexto, los elementos de capital social considerados, fueron: relaciones de grupos y redes (interconexión entre miembros de la empresa) y reciprocidad (retroalimentación y fortalecimiento de las relaciones, en función del nivel de confianza entre miembros) (Durston, 2002).

a. Relaciones de grupos y redes

El análisis de la relación de grupos y redes en la empresa familiar, se centró en el tipo de relación entre grupos, enfocando de manera concreta la sociabilidad entre miembros de la familia y empleados, como mecanismo de capital social para el ascenso dentro de la empresa. En este sentido, todas las empresas familiares consultadas $(100 \%$ de la muestra) evalúan la experiencia laboral y los cargos vacantes para hacer la reasignación de funciones y responsabilidades.

En la mayoría de los casos, predomina la valoración de la experiencia laboral, en términos de buscar el bienestar y satisfacción de los clientes al realizar sus compras. Igualmente se pretende la eficiencia y eficacia en el personal contratado para el desarrollo y crecimiento de la empresa, agilizar las actividades diarias y evaluar las posibilidades de ascensos y sustituciones en vacantes de mayor responsabilidad. Por lo cual se evidencia 
una clara relación empleador-empleado, en la cual no influye de manera determinante el grado de sociabilidad existente entre partes a la hora de decidir el ascenso o la contratación de nuevo personal.

Sobre este particular es importante destacar que las empresas familiares son entidades dinámicas, y en función de su madurez, el número de personas involucradas en cada área aumenta $y$, con ello, se potencia el grado de complejidad en cuanto a sus relaciones (Pérez, 2004). De esta manera, las necesidades del personal se hacen más divergentes y dificultosas de manejar, conduciendo a que la forma de determinar quién debe o no ocupar un cargo sea una tarea de expertos conocedores de las áreas de la empresa.

De esta manera, en la investigación no se evidenció la predominancia de las relaciones personales o de los lazos de amistad establecidos entre los grupos y redes de las empresas familiares consultadas, a la hora de seleccionar o promover al personal; más allá de las relaciones de consanguinidad entre familiares pertenecientes a dichas empresas y en las cuales, como ya se detalló, es claro la línea de mando y el proceso de sucesión.

\section{b. Reciprocidad}

Las empresas consultadas comparten el hecho de que la eficiencia y eficacia en los empleados se mide de acuerdo al desempeño y cumplimiento cabal de las actividades asignadas, así como la habilidad y creatividad que tengan para desempeñarlas. Asimismo, el $25 \%$ de éstas manejan las relaciones de comunicación, superación y participación en la empresa directamente desde la gerencia.
Otro $25 \%$ concede flexibilidad para realizar actividades complementarias, entre ellas académicas, asignando horarios laborales en distintos turnos y permitiendo así la formación y capacitación del personal. Por otro lado, un 50\% no aplica manejo de relaciones de motivación con el personal, según la información suministrada, el personal se limita a cumplir con sus funciones: cada quien en lo suyo, no se puede estudiar y trabajar a la vez (opinión de un empresario entrevistado).

Desde otra perspectiva, el $50 \%$ de los sujetos consultados asume que tanto trabajadores como miembros familiares se interesen por el funcionamiento de la empresa, participando y colaborando cuando es necesario en actividades ajenas a las naturales según el cargo ejercido. El otro $50 \%$ considera que el compromiso se muestra a través del interés del personal para mantener o aumentar las ventas y de esta manera la empresa pueda desarrollarse económicamente.

Igualmente, en el $50 \%$ de las empresas entrevistadas, el personal presta colaboración en actividades diferentes al cargo ejercido. El otro $50 \%$ piensa que el personal debe permanecer en las actividades asignadas, velar por su cargo, demostrar rendimiento en el área donde se desempeña, considerando que los miembros de familia deben ser los jefes.

En este contexto, todas las empresas estiman que a los empleados se les respeta las funciones ejercidas según el cargo. Las responsabilidades son distribuidas equitativamente de acuerdo al trabajo a realizar en el departamento al cual estén adscritos. Es así como las empresas familiares tienen la ventaja de mantener una estrecha relación, estableciendo 
Capital social en empresas familiares

Jiménez, Magda y Piña Zambrano, Henri

lazos y vínculos familiares entre los miembros que la conforman, de donde nace el sentido de pertenencia y propósito común entre sus integrantes.

En función del planteamiento anterior, en las empresas familiares, sobre todo aquellas de primera generación, el fundador es visto como el padre de familia bajo cuya tutela y responsabilidad se encuentran los miembros de la empresa, sean estos miembros de la familia o no. De allí que frecuentemente se establezcan relaciones de parentesco "ceremonial o ficticio" como muestra del resguardo y protección que la familia ofrece a sus empleados no familiares (Monteferrante, 2006).

Resulta oportuno destacar, que los elementos emocionales y afectivos observados en las empresas familiares, son intangibles, sin embargo pueden concretarse en cualidades como el compromiso, confianza, flexibilidad, visión de largo plazo u orgullo, entre otros. Esto conduce a una trascendencia en la relación laboral, una fuerza moral no sólo percibida en los familiares, sino extendida a todos los trabajadores.

Igualmente es importante e resaltar, que en la investigación se evidencio un aspecto particular referente a las nacionalidades. En dos casos donde los miembros familiares son oriundos de la misma nación, predominó la centralización como eje de la empresa. Las decisiones son tomadas desde la gerencia para posteriormente ser comunicadas a los subordinados, no hay participación de estos últimos en los asuntos laborales.

En contraparte, en los otros dos casos se evidenció que la pasión expresada por los miembros de la familia, es transmitida de alguna forma a los empleados no familiares, induciendo un compromiso moral y leal hacia la organización, aspecto éste, poco usual en otros tipos de organizaciones.

\subsection{Funcionamiento de la dinámica de gestión del capital social}

Este aspecto hace referencia al comportamiento colectivo relacionado entre sí, que va desde la sociabilidad (asociatividad) desplegada en las redes sociales hasta el poder expresado en la capacidad política de movilizar efectivamente los recursos del grupo y del nivel de organización con que el grupo resuelve y coordina sus acciones y actividades (Artier, 2004). Destaca en este sentido, la cooperación (trabajo en conjunto) y relaciones familiares (dicotomía familia-empresa).

\section{a. Cooperación}

En el $50 \%$ de las empresas familiares la comunicación entre subordinados y superiores se realiza a través de la comunicación directa, ya sea para manejar conflictos o para manifestar algunas sugerencias. En el otro $50 \%$ la comunicación se canaliza a través de las distintas jerarquías, sin embargo, si la situación es de tratamiento particular, la gerencia se hace responsable directamente del caso.

Todos los miembros de las empresas realizan juntas familiares para llevar a cabo conversaciones relacionadas con su desarrollo y crecimiento, de manera de tomar decisiones, así como para el manejo de conflictos o cuando hay circunstancias que ameriten decisiones entre estos. En un $75 \%$ los empleados y miembros de las empresas muestran interés y atención por su crecimiento. Por su parte, 
un $25 \%$ mantiene el argumento que cada quien debe permanecer en su área de trabajo y velar para por el aumento de las ventas. De acuerdo con estas premisas, en las empresas familiares se manejan los canales de comunicación con los subordinados, bajos distintos formatos y a través de los niveles jerárquicos.

En este sentido, uno de los retos más difíciles en las empresas familiares, es lograr una comunicación eficaz entres su miembros, principalmente cuando se pueden mezclar las relaciones propias de familia con las profesionales y laborales. Para ello, considera que la supervivencia de la organización debería ser la principal obligación de quienes están al frente de ella, por tanto el consejo o junta familiar debe ser el órgano a través del cual la familia se reúna periódicamente con el propósito de debatir aspectos que vinculen a la empresa y a la familia (Vainrub y Rodríguez, 2009).

En la investigación se constató, que las empresas realizan juntas de familia para tomar decisiones concernientes al crecimiento de la organización y a su manejo. Para la consolidación de tales juntas, se debe establecer claramente los puntos de vista de cada miembro así como sus aspiraciones y preocupaciones. Esta será la forma de proyectar ante sus subordinados no familiares el sentido de compromiso, respeto, valores y pertenencia hacia la labor desempeñada y hacia la empresa, y de esta manera, fortalecer el compromiso moral de éstos, aunque sean ajenos a la familia.

\section{b. Relaciones familiares}

Las empresas consultadas afirma que aún no se ha producido despidos o re- nuncias por parte de miembros de la familia, pero en caso de suceder, se procederá de manera legal según el cargo y acciones que posea en la organización y por la vía del dialogo. De igual forma se comprobó, que las empresas familiares aparece una junta directiva como máximo órgano encargado de dirigir y controlar la organización, también, en dos de los casos estudiados esta dirección es unipersonal.

Por otro lado, el $75 \%$ de las empresas, prevé que la gerencia de ésta continúe en manos de familiares y mantener esa figura jurídica. Por su parte, el $25 \%$ desconoce quién será el responsable de la empresa en el futuro, puesto que se encuentran concentrados en hacer crecer la empresa bajo la actual administración de los miembros familiares, al frente de ella.

En este sentido, el $75 \%$ de los consultados tiene previsto capacitar al sucesor de la empresa en áreas que le permita dirigirla y conducirla, para continuar manteniendo la figura de empresa familiar. El 25\% considera que deben conocer de gerencia quienes este llevando las riendas de la empresa.

Se observa claramente, que a pesar de las debilidades que puedan tener las empresas familiares, la lucha por permanecer bajo esta figura es constante, el interés por mantener el control legal sobre la propiedad, la dirección y el control financiero es una preocupación que se traslada de generación en generación. Asimismo, pudo percibirse el planteamiento realizado en las juntas familiares, respecto que sean los hijos quienes se sean los responsables de la empresa en el futuro, de acuerdo con su experiencia y manejo administrativo de la organización. 
Capital social en empresas familiares

Jiménez, Magda y Piña Zambrano, Henri

En este sentido, la concepción de los empresarios en relación a la sucesión en las empresas familiares, está vinculada con la muerte de los fundadores, mientras el fundador va supuestamente declinando, los hijos van creciendo: son curvas que no siempre se cortan simétricamente en el tiempo. La sucesión debe ser considerada como el relevo de un cargo, para el cual hay que formarse y adquirir experiencia en cuanto a la plataforma administrativa sobre la cual se rige (Bustillo, 2006).

Es evidente, que las juntas familiares juegan un papel importante en las tomas de decisiones y en las relaciones familiares de la empresa, de ellas pueden surgir medidas para conocer quién será el responsable del negocio una vez que el fundador no esté o decida delegar funciones por diversas razones. Finalmente, La sucesión de los bienes familiares debe ser vista con antelación. El más capacitado de los miembros, es quien debería asumir el liderazgo de la empresa, siempre y cuando sea aprobado por todos los accionistas (Bustillo, 2006).

De acuerdo con el análisis realizado, las empresas familiares se corresponden con un tipo de organización que en la actualidad está consolidándose en el mundo empresarial, su manejo y permanencia en el tiempo depende directamente del conocimiento y capacidad para su administración, control y dirección.

Igualmente es un aspecto resaltante en el desarrollo y crecimiento de las empresas familiares, el compromiso, sentido de pertenencia y confianza por fortalecerla.

Las empresas familiares han tomado tal importancia en la ciencia económica que ya se habla de una línea com- pleta de especialización donde no sólo se estudian las situaciones empresariales como la planeación de la nueva generación, sino que es conveniente familiarizar a los futuros sucesores con su desempeño interno, políticas y su modo de funcionar, proveerles de experiencias, tanto dentro de la compañía como fuera de ella.

Para lograr que una empresa familiar sea exitosa, se considera necesaria la capacidad de dirigir y disciplinar a la familia de forma tal que su comportamiento natural esté alineado con el manejo profesional del negocio.

Es allí entonces, donde se distingue el capital social como elemento intangible característico del buen funcionamiento en las organizaciones, generando ambientes propicios para la confiabilidad de las relaciones promotoras de los flujos comunicacionales y de relaciones laborales, promotores de la colaboración, cooperación, asociatividad y confianza en la realización y cumplimiento de las tares en la empresa.

Es de resaltar asimismo, que estos elementos del capital social, deben funcionar como mecanismos para permitir a los miembros de familia en sus empresas, despertar en los empleados no familiares el sentido de interés por su buen funcionamiento y crecimiento empresarial.

En función del análisis anterior, puede concluirse que en la dinámica presente en las empresas familiares del estado Falcón, resaltan elementos de capital social tal como la colaboración entre los diferentes grupos del colectivo humano que hace vida en la organización, basadas en la confianza, las normas efectivas, valores y las redes sociales. 
En este sentido, las características de la dinámica del capital social en empresas familiares del estado Falcón, se centran en las normas y las redes que permiten a los empleados actuar de manera colectiva en las distintas actividades.

De igual forma, se visualiza el capital social en el conjunto de relaciones, actitudes y valores que determinan las interacciones entre los trabajadores y los miembros de la familia, a la vez, una red social que produce utilidades y beneficios para el colectivo que participan de las labores de la organización.

\section{Conclusiones}

Si bien las empresas familiares sujeto de esta investigación, aplican criterios de gestión empresarial los cuales les permiten la conducción y dirección de la organización en función de los intereses familiares, esta tarea requiere de más atención en cuanto al diseño de estrategias y estructuras para abordar el mercado, de tal manera que el legado pueda pasar de una generación a otra.

En este sentido, la planificación y los métodos de trabajo son uno de los aspectos que usualmente deben prestar mayor interés en las empresas familiares, de acuerdo con las responsabilidades asumidas con los empleados y visto que el compromiso y lealtad de éstos últimos buscan generalmente establecer relaciones de largo plazo con los miembros de la familia.

Así, la sociabilidad y la confianza entre miembros de la familia y empleados conduce a establecer lazos de pertenencia de desarrollo y crecimiento, dada la certidumbre y la estabilidad de la empresa familiar, ésta inspira en los empleados un sentimiento de orgullo que redunda en mayor compromiso y dedicación a sus actividades, con la finalidad de enfatizar las relaciones entre jefes y subordinados.

Por otro lado, las empresas familiares representan hoy día una fuerte figura jurídica causante de impacto y desarrollo económico a nivel empresarial, por tanto es importante dar continuidad a proyectos como este estudio, con la finalidad de identificar describir y analizar la realidad de las organizaciones y distinguir cuál debe ser la forma correcta o la más idónea para llevar a cabo la conducción y gerencia de ellas, sin tener que llegar a los extremos de dejar morir a las empresas por desconocimiento de sus manejos y administración.

En este sentido es muy propicio, el establecer vínculos con la Universidad Nacional Experimental Francisco de Miranda (UNEFM), y a través del programa Desarrollo Empresarial, para obtener conocimiento teórico en relación a los elementos a tener presentes en la conducción y gerencia de una empresa y posteriormente ejecutarlos, lo cual permitiría un impulso positivo al crecimiento de las organizaciones.

Asimismo, es importante mantener en lo posible, relaciones familiares armoniosas, de tal manera que se consoliden las empresas familiares a través de la aplicabilidad del capital social como elemento intangible que se convierta en compromiso, responsabilidad, valores, respeto y sentido de pertenencia por el trabajo realizado y cumplimiento cabal de las tareas laborales. 
Capital social en empresas familiares

Jiménez, Magda y Piña Zambrano, Henri

\section{Referencias bibliográficas}

Arriagada, Irma (2006). Breve guía para la aplicación del enfoque de capital social en los programas de pobreza. Santiago de Chile. Comisión Económica para América Latina (CEPAL). 54 pp.

Artier, José (2004). Problemas de gestión en la empresa familiar. Documento en línea. Extraído 30 de Noviembre, 2009 de la dirección electrónica http://www. Cseco.org/fr/pdf/ml/spanish/Spanish problems_in_managing_a_familyowned_business.pdf

Beltrán, Jesús (2004). Indicadores de gestión: guía práctica para estructurar acertadamente estas herramientas clave para el logro de la competitividad. 3R editores. Colombia. 147 pp.

Bourdieu, Pierre (1985). The social space and the genesis of groups. Theory and Society, 14 (6): 723-744.

Bourdieu, Pierre (1989). Social space and symbolic power. Sociological Theory, 7 (1): 14-25.

Bustillo, J. (2006) Empresas familiares: Encuentro de dos mundos. Debates IESA, XI (2): 22-26.

Coleman, James (1986). Social theory, social research and a theory of action. The American Journal of Sociology, 91 (6): 1309-1335.

Coleman, James (1990). Social institutions and social theory. American Sociological Review, 55 (3): 333-339.

Coleman, James (1992). The problematics of social theory: four reviews of foundations of social theory. Theory and Society, 21 (2): 263-283.

Contreras, Ricardo y Regalado Rafael (2009). Potencialidades y problemas en empresas de participación familiar. Ediciones Universidad de Málaga. España. 79 pp.
Durston, John (2002). El capital social campesino en la gestión del desarrollo rural. Díadas, equipos, puentes y escaleras. Santiago de Chile. Comisión Económica para América Latina y el Caribe (CEPAL). 168 pp.

Flores, María (1999). Análisis de la estructura organizacional de una cadena de supermercado. Guatemala. Universidad de Marroquín. 98 pp.

Forsman, María (2005). Development of research network: the case of social capital. Helsinki: Abo Akademi University Press. 238 pp.

Fukuyama, Francis (1999). Social capital and civil society. Washington. International Monetary Fund. IMF Conference on Second Generation Reforms.

Granovetter, Mark (1973). The strength of weak ties. American Journal of Sociology, 78 (6): 1360-1380.

Granovetter, Mark (1985). Economic action and social structure: the problem of embeddedness. American Journal of Sociology, 91 (3): 481-510.

Hanifan, Lyda (1916). The rural school community. Annals of the American Academy of Political Sciences, 67: 130-13.

Kliksberg, Bernardo (2001). El Capital Social. Editorial Panapo. Caracas. 150 pp.

López, Alejandra y Contreras, Ricardo. 2009. Análisis sobre capital social, cultural y simbólico en las empresas tradicionales de Celaya: Fabrica de cajeta y dulces. Contribuciones a las Ciencias Sociales, Extraído el 30 de enero, 2010 de www.eumed.net/rev/cccss/04/cgcg.htm

Ministerio de Economía (ME) (2003). Guía para para la pequeña y mediana empresa familiar. Madrid. Dirección General de Política de la Pequeña y Mediana Empresa. 119 pp.

Milani, Carlos (2003). Social capital and local development theories: lessons 
from the Pintadas experience. Bahia (Brasil). Escola de Administração at the Federal University of Bahia. 31 pp.

Moreno, José (2005) Memorías del Seminario Internacional: "Capital Social en Acción". Universidad Metropolitana, Caracas, Venezuela, 6 de abril. pp.137.

Monteferrante, Patricia (2006) Empresas familiares: ¿Pulperías o empresas profesionales? Debates IESA, XI (2): 1216.

Pérez, Rafael (2004) Propuesta de un modelo de gestión humana y cultura organizacional para PyMES innovadoras, Revista EAN, 47, 46-65.

Puente, Eva y Torrella, Roger (2003). Social capital as a managerial phenomenon. Tampere (Finlandia): Tampere University of Technology. Department of industrial engineering and management. $63 \mathrm{pp}$.
Putnam, Robert (1995). Bowling alone: America's declining social capital. Journal of Democracy, 6 (1): 65-78.

Putnam, Robert (2003). Work and social capital. Saguaro Seminar on Civic Engagement in America. Nueva York. 16 pp.

Putnam, Robert (2004). Education, diversity, social cohesion and social capital. Meeting of OECD Education Ministers (págs. 2-7). Dublín. OECD.

Tocqueville, Alexis de (1854). La democracia en América. Madrid. José Trujillo.

Vainrub, Roberto y Rodríguez, Aramis (2009). Empresas familiares: sinergia o desastre. Debates IESA, X (3): 30-32.

Vainrub, Roberto (2006). Convertir sueños en realidades. Una guía práctica para emprendedores. Ediciones IESA. Caracas. 165 pp. 